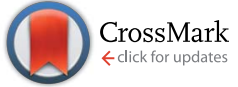

Cite this: RSC Adv., 2015, 5, 35346

Received 30th January 2015

Accepted 1st April 2015

DOI: $10.1039 / c 5 r a 01685 a$

www.rsc.org/advances

\section{Precise control of nanoparticle surface by host- guest chemistry for delivery to tumor $\dagger$}

\author{
Hisato Matsui, ${ }^{a}$ Motoki Ueda, ${ }^{\text {I }}$ Isao Hara ${ }^{\mathrm{c}}$ and Shunsaku Kimura*a
}

Nanoparticles were prepared by host-guest chemistry using stereo-complex formation between righthanded and left-handed helical peptides. The host molecule is a 3rd generation polyamidoamine dendrimer having 16 terminated right-handed helices. Three types of guest molecules were examined: poly(sarcosine)- $b$-(D-Leu-Aib) 6 (AB-LP), (poly(sarcosine) $)_{3}-b$-(D-Leu-Aib) 6 (A 3 B-LP), and (D-Leu-Aib) 6 - $b-$ (poly(sarcosine $))_{3}\left(A_{3} B\right.$-apLP). All the guest peptides associate stoichiometrically with the host dendrimer because of the stereo-complex formation. When $A_{3} B$-apLP associates with the host dendrimer, the conjugate shows a hydrodynamic diameter of $27 \mathrm{~nm}$, which is explainable by the fact that 16 guest peptides are incorporated in the host dendrimer with tight helix packing and an antiparallel helix dipole arrangement. The nanoparticles were labeled with indocyanine green fluorescence agent and were applied for tumor imaging. Among them, the conjugate with $A_{3} B$-apLP shows a long life time in the blood stream and a good tumor/liver signal ratio. Furthermore, the conjugate does not trigger the accelerated blood clearance phenomenon. Although these nanoparticles that were modified by similar guest molecules should have similar surfaces, their in vivo disposition is significantly affected.

\section{Introduction}

Nanoparticles have been attracting considerable attention in the field of theranostics, ${ }^{1}$ which is currently considered to be essential for personalized medicine. ${ }^{2-5}$ They can load various types of imaging agents and therapeutic agents. Nanoparticles loading imaging probes can provide information on in vivo disposition, which can determine appropriate doses for individuals and can predict adverse effects of the nanoparticle loaded drugs. There is, however, a serious obstacle for nanoparticles to be used as a vehicle for imaging and therapeutic agents, which is the pharmacokinetic change of the nanoparticles between the first dose of the imaging nanoparticle and the following dose of the same nanoparticle or the therapeutic nanoparticle. Pharmacokinetic alterations can be caused by two reasons. One is the change in the physical properties (particle size, structural stability, surface density, thickness of hydrophilic shell, and half-life time in blood stream) of the nanoparticle upon labelling and loading different agents for the diagnosis and therapy on the nanoparticle. The other is the immune response to the nanoparticle, which is known as the

${ }^{a}$ Department of Material Chemistry, Graduate School of Engineering, Kyoto University, Kyoto-Daigaku-Katsura, Nishikyo-ku, Kyoto, 615-8510, Japan. E-mail: shun@scl. kyoto-u.ac.jp

${ }^{b}$ Clinical Division of Diagnostic Radiology, Kyoto University Hospital, 54 Shogoin Kawara-cho, Sakyo-ku, Kyoto, 606-8507, Japan

${ }^{c}$ Technology Research Laboratory, Shimadzu Corporation, Kyoto 619-0237, Japan

$\dagger$ Electronic supplementary information (ESI) available. See DOI: 10.1039/c5ra01685a accelerated blood clearance (ABC) phenomenon. ${ }^{6-9}$ In order to solve these difficulties, it is imperative to examine various types of the nanoparticles, and thus we prepared nanoparticles using host-guest chemistry.

One typical example of nanoparticles is a polymeric micelle. ${ }^{10}$ It is easy to load hydrophobic agents at the hydrophobic core of core-shell type micelles and to insert functional polymers via the hydrophobic interaction. However, size control is a difficult task because of the swelling of the polymeric micelle when loading these agents ${ }^{\mathbf{1 0}}$ or changing the hydrophilic-hydrophobic balance by binding functional molecules. Furthermore, the modification of nanoparticle surfaces with a diagnosis probe affects their life time in the blood stream. ${ }^{\mathbf{1 1}}$ With the aim to solve these problems, we propose a novel molecular assembly using host-guest chemistry applied to a dendritic host scaffold, which makes it possible to construct one nanoparticle and keep a defined number of the constituent amphiphilic polymers and the core size unchanged upon the incorporation of functional groups. Accordingly, the dendritic core has eight amphiphilic polypeptides as a host molecule to incorporate eight amphiphilic polypeptides as a guest molecule. ${ }^{12}$ These polypeptides have a helical hydrophobic block with opposite helicity, and these two types of helices form a stereo-complex with a $1: 1$ stoichiometry. ${ }^{12-15}$ We successfully prepared a polymeric nanoconjugate containing sixteen amphiphilic polypeptides using host-guest chemistry. With the chemical modification of the guest amphiphilic polypeptide by a diagnostic or therapeutic agent, this type of nanoconjugate can therefore be functionalized by keeping the size unchanged 
a
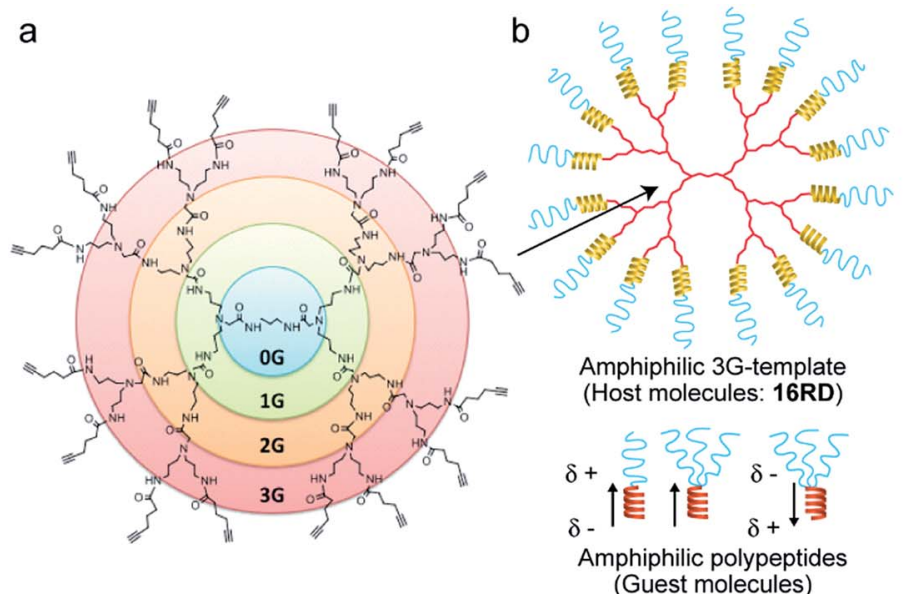
Guest molecules)

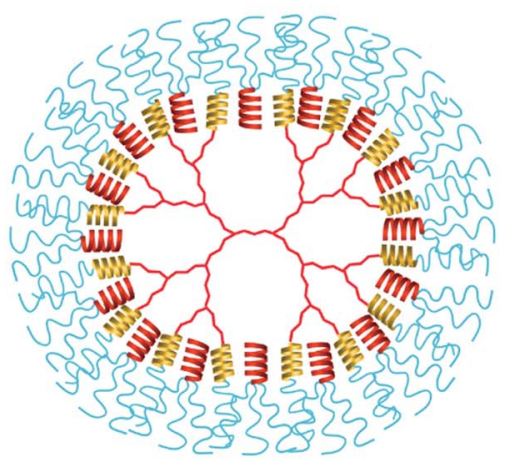

Conjugate

C

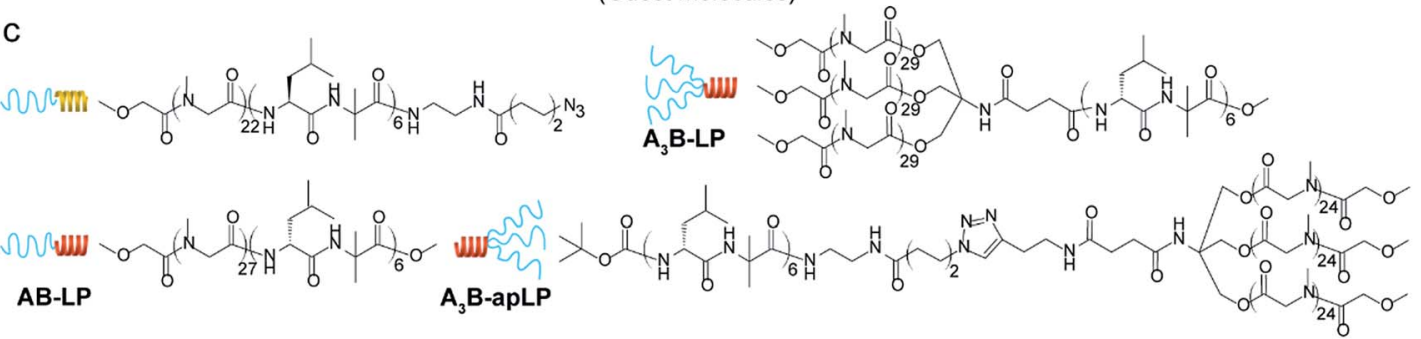

Fig. 1 Schematic illustration and chemical structure of host and guest molecules. Schematic illustration of the 3rd generation dendrimer core (a), amphiphilic dendrimer template, three amphiphilic guest molecules and host-guest nanoconjugate (b). Chemical structure of amphiphilic three guest molecules (AB-LP, $\left.A_{3} B-L P, A_{3} B-a p L P\right)$ (c).

due to the constant core size of the dendritic host molecule and the defined number of guest peptides. Here, we extended this scheme to a dendrimer with sixteen amphiphilic polypeptides in combination with three types of the guest amphiphilic polypeptides to achieve more precise molecular control of the nanoparticle (Fig. 1). In order to evaluate the nanoparticle properties precisely, we also examined the availability of the nanoparticle for tumor imaging.

\section{Results and discussion}

\section{Self-assembly by host-guest chemistry}

The association of the guest peptides (AB-LP, $\mathrm{A}_{3} \mathrm{~B}-\mathrm{LP}$ and $\mathrm{A}_{3} \mathrm{~B}-$ apLP) with the host dendrimer (16RD) was analyzed by dynamic light scattering (DLS) measurements by varying the feed molar ratios of the guest peptides against the host. In these combinations, there were three types of molecular assemblies coexisting in the mixtures, the assemblies of the pure guest, the pure host, and a mixture of the guest and the host, because these guests and the host are amphiphilic by themselves. The former two types of molecular assemblies became relatively large having size over $200 \mathrm{~nm}$. The guest molecules alone formed a curved sheet of $c a .200 \mathrm{~nm}$ square as previously reported. ${ }^{12,16-19}$ The host took on a small disk-like structure, which quickly grew into larger aggregates of $c a .200 \mathrm{~nm}$ size by itself, because the peripheral sixteen poly(sarcosine) chains of the host cannot shield the hydrophobic blocks inside. ${ }^{12}$ On the other hand, upon the association of the guest peptide with the host, the molecular assemblies became smaller (Fig. 2).
A mixture of AB-LP and $16 \mathrm{RD}$ at the feed molar ratio of $16: 1$ formed a molecular assembly having a minimum diameter of $49 \mathrm{~nm}$ (Fig. 2a), suggesting that they should associate in a stoichiometric manner of $1: 1$ between the right-handed helices of 16RD and the left-handed helices of AB-LP as expected. With a decrease in the ratios below $16: 1$, the hydrodynamic diameters became larger, up to $200 \mathrm{~nm}$, and

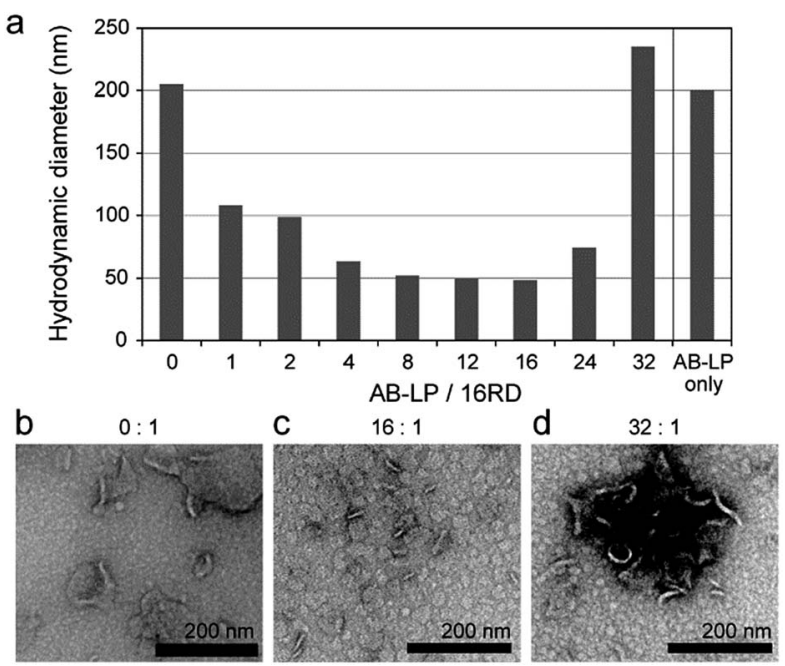

Fig. 2 Hydrodynamic diameters vs. feed molar ratios of AB-LP/16RD by DLS measurement (a) and TEM images (negative staining with uranyl acetate) of molecular assembly prepared from a mixture of AB-LP and 16RD at a molar ratio of $0: 1$ (b), $16: 1$ (c) and $32: 1$ (d). 
this size corresponds to the molecular assembly of pure 16RD. The hydrodynamic diameters also became larger with an increase in the ratios above 16:1 because the excess AB-LP generated curved sheets of $200 \mathrm{~nm}$. Therefore, it is considered that $16 \mathrm{RD}$ cannot accommodate more than $16 \mathrm{~mol}$ equivalents of the guest peptides.

TEM images (Fig. 2b-d) supported the coexistence of these three types of molecular assemblies in the mixtures. Molecular assemblies of about $50 \mathrm{~nm}$ size were formed from a mixture of AB-LP and 16RD in a molar ratio of $16: 1$ (Fig. 2c). In addition to the size of the molecular assemblies, large curved sheets coexisted when AB-LP and 16RD were mixed in a molar ratio of $32: 1$. The mixed solutions were filtered through a cut-off membrane of $20 \mathrm{kDa}$ to remove the molecular assemblies of 16RD or AB-LP with sizes over $200 \mathrm{~nm}$, and the filtrates were subjected to $\mathrm{CD}$ measurements. The Cotton effects due to the remaining helicity of the guest (left-handed helix)-host (righthanded helix) conjugate decreased with an increase in the ratios of the guest up to 16 . Upon further increasing the ratios to 24 and 32, the Cotton effects remained nearly zero (Fig. S2 in ESI $\dagger$ ), which supports the fact that the small conjugate was composed of 16RD and sixteen AB-LPs and that 16RD cannot accommodate more than $16 \mathrm{~mol}$ equivalents of the guest peptides.

The hydrodynamic diameter of $49 \mathrm{~nm}$, however, is significantly larger than the estimated value of $c a .25-30 \mathrm{~nm}$ for the guest-host conjugate. TEM observations revealed that the guest-host conjugates aggregated to some extent in a timedependent manner. It is therefore considered that the surface property of the $\mathrm{AB}-\mathrm{LP} / 16 \mathrm{RD}$ conjugate was not hydrophilic enough to shield the hydrophobic helix layer of the conjugate. Indeed, the morphology of the conjugate was found to be disklike, which has been frequently observed with lower generation dendrimers due to the low density of the dendritic chains inside. Therefore, in order to suppress the aggregation of the conjugate, we designed $\mathrm{A}_{3} \mathrm{~B}$-type guests.

The hydrodynamic diameters of the $\mathrm{A}_{3} \mathrm{~B}$-type guest peptides/ host (16RD) conjugate were measured by varying the ratios of the guest against the host. The minimum hydrodynamic diameters were obtained with the additions of 16 mol equivalent $\mathrm{A}_{3} \mathrm{~B}$-LP and $14 \mathrm{~mol}$ equivalent $\mathrm{A}_{3} \mathrm{~B}$-apLP to be $32 \mathrm{~nm}$ and 27 $\mathrm{nm}$, respectively (Fig. S3 in ESI $\dagger$ ). Thus, these guest peptides also associated stoichiometrically with the host dendrimer on the basis of the stereo-complex formation between the righthanded and the left-handed helices. Furthermore, at mixing ratios of $8: 1,12: 1$ and $14: 1$, the guest-host associates also maintained a similar size (Fig. S3†), suggesting that the dendrimer core should be a primary determinant of the associate sizes. TEM observation revealed that the conjugates still maintained the disk-shaped morphology based on the wormlikeshaped side-view and the sphere-shaped top-view (Fig. 3), but no aggregation was detected at room temperature for $24 \mathrm{~h}$. The local high density of poly(sarcosine) chains of the $\mathrm{A}_{3} \mathrm{~B}$-type peptides should provide sufficient hydrophilicity. Not sphereshaped, but a disk-shaped assembly may be obtained because the $3 \mathrm{G}$ dendrimer was not sphere-shaped but ellipsoidshaped. ${ }^{20}$

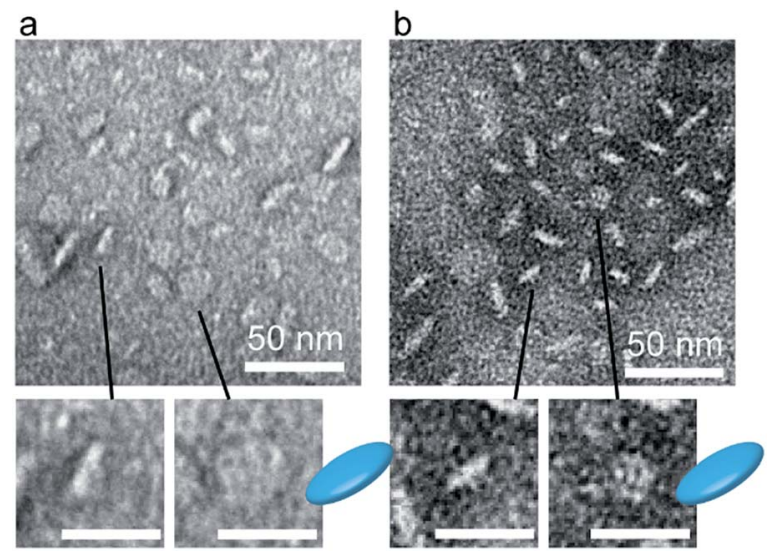

Fig. 3 TEM images of molecular assemblies prepared from a mixture of $\mathrm{A}_{3} \mathrm{~B}-\mathrm{LP} / 16 \mathrm{RD}$ (a) and $\mathrm{A}_{3} \mathrm{~B}-\mathrm{apLP} / 16 \mathrm{RD}$ (b) at the molar ratio of $16: 1$. Magnified figures are shown at the bottom. Scale bars in the magnification are $25 \mathrm{~nm}$.

The hydrodynamic diameter of $27 \mathrm{~nm}$ for the $\mathrm{A}_{3} \mathrm{~B}-\mathrm{apLP} / 16 \mathrm{RD}$ conjugate corresponds just to the estimated diameter where the guest helices and the host helices take on an interdigitated side-by-side arrangement with antiparallel dipole orientation (Fig. 4). On the other hand, the $\mathrm{A}_{3} \mathrm{~B}-\mathrm{LP} / 16 \mathrm{RD}$ conjugate showed a slightly larger diameter of $32 \mathrm{~nm}$, which may be explained by the dipole-dipole interaction, which works to thicken the helical peptide layer in the conjugate as follows. When $A_{3} B-L P$ is inserted into the surface peptide region of $16 \mathrm{RD}, \mathrm{A}_{3} \mathrm{~B}-\mathrm{LP}$ should take an orientation to keep the bulky $\mathrm{A}_{3}$-block outside of 16RD. The helix block of $\mathrm{A}_{3} \mathrm{~B}-\mathrm{LP}$ then prefers a head-to-tail arrangement with the helix block of 16RD in order to avoid a side-byside arrangement, where the parallel arrangement of the helix dipoles should destabilize the helix packing in the conjugate. It is therefore considered that the steric effect in the association of the guest peptide with the host dendrimer should take priority, and the following dipole-dipole interaction should decide the helix packing of the side-by-side or the head-to-tail arrangement in the conjugates (Fig. 4). ${ }^{21}$ Accordingly, as far as the 2 nd generation ${ }^{\mathbf{1 2}}$ and the 3 rd generation dendrimers are concerned, there is no difference in the host-guest chemistry in the present molecular systems.

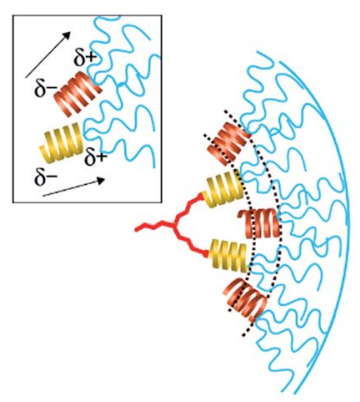

16RD / A B-LP

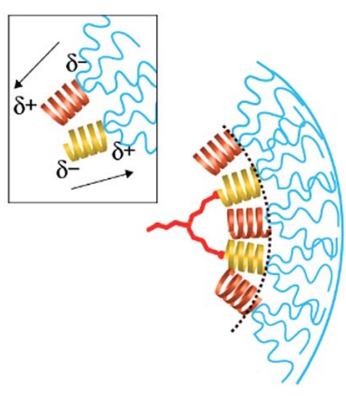

16RD / A B-apLP
Fig. 4 Illustrations of molecular packing in the hydrophobic layer in the case of $A_{3} B-L P / 16 R D$ (left) and $A_{3} B$-apLP/16RD (right). Arrows mean the dipole moments along hydrophobic $\alpha$-helices. 
a
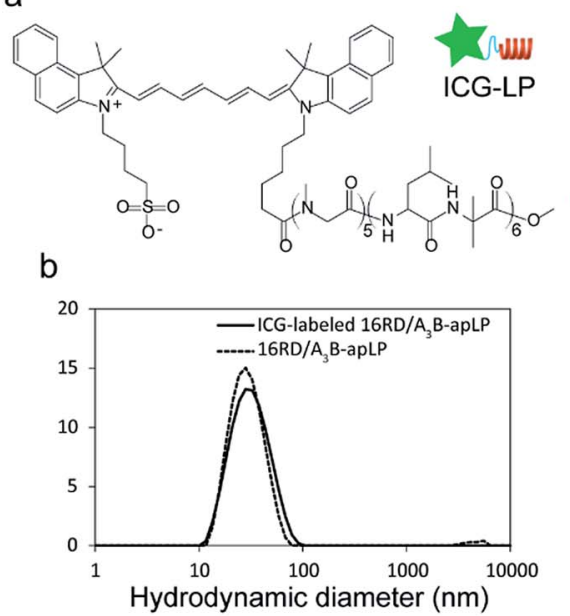

$\mathrm{C}$
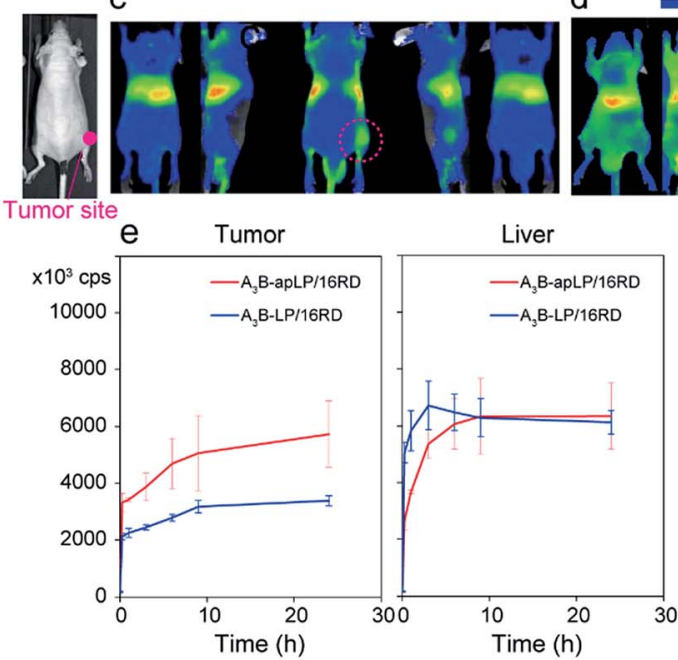

600

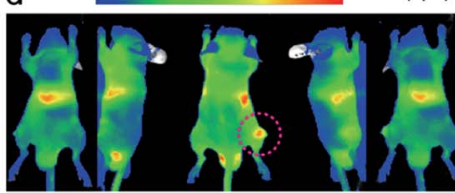

Tumor/Liver

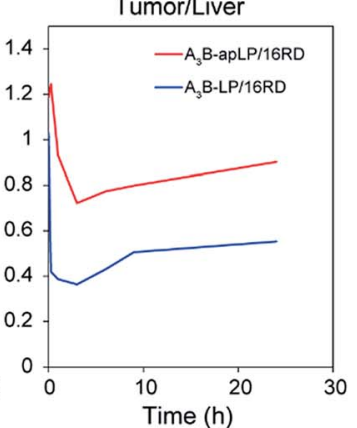

Fig. 5 Chemical structure of ICG-labeled amphiphiles, ICG-LP (a), DLS profiles of molecular assemblies prepared from a mixture of $A_{3} B$-apLP and $16 R D$ at the mixing ratio of $14: 1$ with and without ICG-LP at 0.16 eq. (b) In vivo NIRF imaging results (c-e). NIRF images of nanocarriers from $\mathrm{A}_{3} \mathrm{~B}-\mathrm{LP} / 16 \mathrm{RD} / \mathrm{ICG}-\mathrm{LP}$ (c) and $\mathrm{A}_{3} \mathrm{~B}-\mathrm{apLP} / 16 \mathrm{RD} / \mathrm{ICG}-\mathrm{LP}$ (d) after $9 \mathrm{~h}$ from administration. The region of interest (ROI) at the tumor site, liver site and tumor/liver NIRF intensity ratio (e)

\section{In vivo disposition}

The $\mathrm{A}_{3} \mathrm{~B}-\mathrm{LP} / 16 \mathrm{RD}$ conjugate and the $\mathrm{A}_{3} \mathrm{~B}$-apLP/16RD conjugate have the same constituents except for the direction of the helix dipoles of the guest being parallel or antiparallel to that of the host. The structural difference makes the size of the $A_{3} B-L P /$ 16RD conjugate slightly larger than the $\mathrm{A}_{3} \mathrm{~B}-\mathrm{apLP} / 16 \mathrm{RD}$ conjugate. These two types of conjugates were labeled with ICG and examined for tumor imaging of mice. Even though the surface modification between these two conjugates looks very similar, they showed a different behavior for in vivo pharmacokinetics.

A mixture of $16 \mathrm{RD}, \mathrm{A}_{3} \mathrm{~B}$-apLP and ICG-LP (Fig. 5a) at a ratio of $1: 14: 0.16$ generated the ICG-labeled conjugate with a diameter of $27 \mathrm{~nm}$, which was the same size of the $\mathrm{A}_{3} \mathrm{~B}$-apLP/16RD conjugate at a mixing ratio of $1: 14$ (Fig. 5b). This observation is a good evidence for the present guest-host chemistry in the preparation of functionalized nanoparticles, as it is very useful for size control. The buffered solutions of the ICG-labeled conjugates were injected to tumor bearing mice from the tail vein and NIRF images were taken by a Shimadzu Clairvivo OPT (Fig. 5). When the images that were taken $9 \mathrm{~h}$ after the injection were compared, the whole body fluorescence intensity was significantly larger with the $\mathrm{A}_{3} \mathrm{~B}-\mathrm{apLP} / 16 \mathrm{RD}$ conjugate than the $A_{3} B-L P / 16 R D$ conjugate, reflecting that the circulating amount of the $\mathrm{A}_{3} \mathrm{~B}$-apLP/16RD conjugate in the blood stream was larger than that of the $A_{3} B-L P / 16 R D$ conjugate (Fig. $5 c$ and $d$ ). This is because the $A_{3} B-L P / 16 R D$ conjugate is initially more easily captured by the liver to reduce its concentration in the blood stream, as shown in Fig. 5e. As a result, the amount of accumulation of the $\mathrm{A}_{3} \mathrm{~B}$-apLP/16RD conjugate in the tumor was nearly two-times higher than that of the $\mathrm{A}_{3} \mathrm{~B}-\mathrm{LP} / 16 \mathrm{RD}$ conjugate. The tumor/liver signal ratio is also better with the $\mathrm{A}_{3} \mathrm{~B}$ apLP/16RD conjugate than the $\mathrm{A}_{3} \mathrm{~B}-\mathrm{LP} / 16 \mathrm{RD}$ conjugate (Fig. 5e). The stability of the $A_{3} B-a p L P / 16 R D$ conjugate in the blood stream may be attributable to the 1.3-times higher surface density of the poly(sarcosine) chains with the $\mathrm{A}_{3} \mathrm{~B}-\mathrm{apLP} /$ $16 R D$ conjugate than the $A_{3} B-L P / 16 R D$ conjugate on the basis of a simple calculation of the surface areas of the diameters of 27 $\mathrm{nm}$ and $32 \mathrm{~nm}$.

Furthermore, the antiparallel packing of the helix dipoles in the $A_{3} B-a p L P / 16 R D$ conjugate may contribute to the physical stability in the blood stream. The physical stability is indeed supported by the observation that the amount of accumulation in the tumor site increased with time up to $20 \mathrm{~h}$, which means that the ICG-LP stayed stably in the conjugate in the blood stream.
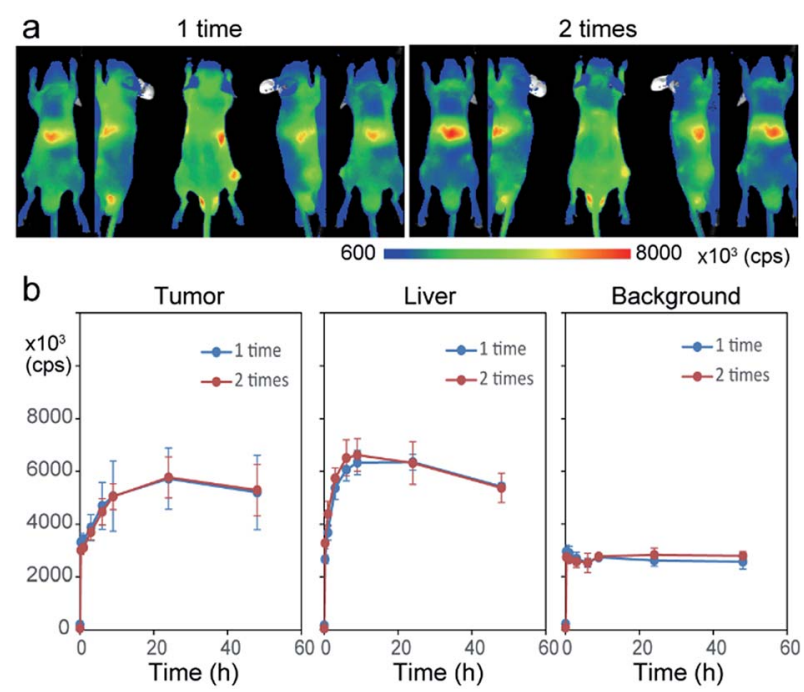

Fig. 6 NIRF images of nanocarrier from $A_{3} B$-apLP/16RD/ICG-LP after $9 \mathrm{~h}$ from 1st and 2nd administrations (a) and the time profiles of ROI at the tumor site, liver site and background (left-leg site) (b). 
The $\mathrm{A}_{3} \mathrm{~B}-\mathrm{apLP} / 16 \mathrm{RD}$ conjugate was examined for in vivo pharmacokinetics upon repeated administrations. It has been reported that the PEGylated liposome and the polymeric micelles at the second dose were immediately captured by liver even though they showed a long life time in the blood stream at the first dose. ${ }^{6-9}$ This type of alteration in pharmacokinetics is called the accelerated blood clearance (ABC) phenomenon. When the $A_{3} B$-apLP/16RD conjugate was dosed at 7 days after the first injection, the time-profiles of the accumulation in the tumor, liver, and background coincided just with those at the first dose (Fig. 6). The reason for no ABC phenomenon with the $\mathrm{A}_{3} \mathrm{~B}$-apLP/16RD conjugate is yet to be solved, but this type of the conjugate should be useful as a nanocarrier platform for clinical tumor diagnostics and therapy, which can be attained by loading the diagnostic or therapeutic agent on the guest peptide. With this platform, nanoparticles can keep their size upon functionalization without an immune response on multiple administrations.

\section{Conclusions}

We demonstrated a useful nanocarrier whose diameter is less than $30 \mathrm{~nm}$. Various chemical agents can be loaded on the nanocarrier with a defined concentration and keeping the size unchanged. The nanocarrier showed a long life time in the blood stream, and no ABC phenomenon was triggered. Tumor imaging is available with the nanocarrier due to the enhanced permeability and retention effect. The chemistry of the nanocarrier is based on the stereocomplex formation between the right-handed and the left-handed helices. The 16 guest peptides can be accommodated in the 16-helix terminated dendrimer due to the stereo-complex formation. The surface of the conjugate is densely covered with poly(sarcosine) chains due to its $A_{3} B$-type molecular architecture. The conjugate was highly stable in the blood stream because of the tight helix packing and antiparallel arrangement of the helix dipoles in the conjugate. We tried functionalizing the conjugate using an ICGmodified guest peptide for tumor imaging. We think various functionalization will be available with just changing the ICG moiety with other agents, by which the nanoparticle size and the long life time in the blood stream will be kept unchanged, and noticeably no $\mathrm{ABC}$ phenomenon of the conjugate nanoparticle will be induced.

\section{Experimental section}

\section{Materials and methods}

Dendrimer (host molecule) and amphiphilic helical peptides (guest molecules). The 3rd generation (3G) PAMAM dendrimer template (16RD) and three types of amphiphilic polypeptides of $\mathrm{Sar}_{27}-b$-(D-Leu-Aib) 6 (AB-LP), (Sar 29$)_{3}-b$-(D-Leu-Aib) ${ }_{6}\left(\mathrm{~A}_{3} \mathrm{~B}-\mathrm{LP}\right)$, and (D-Leu-Aib) $)_{6}-b$-( Sar $\left._{24}\right)_{3}$ (A A $_{3}$-apLP) (Fig. 1) were synthesized in accordance with the ESI $\dagger$ and previous studies..$^{10,16-18}$ 16RD (16 right-handed-helices-modified dendrimer) was synthesized using a click reaction between the 16 terminal azido groups of the dendrimer and the acetylene group at the C-terminal of the right-handed amphiphilic polypeptide. AB-LP (AB-type left- handed helical peptide) has one hydrophilic poly(sarcosine) chain (A) and one left-handed helical hydrophobic peptide (B). On the other hand, $\mathrm{A}_{3} \mathrm{~B}$-LP $\left(\mathrm{A}_{3} \mathrm{~B}\right.$-type left-handed helical peptide) and $\mathrm{A}_{3} \mathrm{~B}$-apLP ( $\mathrm{A}_{3} \mathrm{~B}$-type anti-parallel left-handed helical peptide) are composed of three poly(sarcosine) chains $\left(\mathrm{A}_{3}\right)$ and one left-handed helix (B). The structural difference between $\mathrm{A}_{3} \mathrm{~B}$-LP and $\mathrm{A}_{3} \mathrm{~B}$-apLP is that three poly(sarcosine) chains are attached at either $\mathrm{N}$-terminal or the $\mathrm{C}$-terminal of the hydrophobic helix peptide. Poly(sarcosine) was used due to its thicker polymer chain than poly(ethylene glycol), which contributes to the formation of a dense hydrophilic layer around the molecular assemblies. The syntheses of all compounds were confirmed by ${ }^{1} \mathrm{H}$ NMR and MALDI-TOF MASS analyses.

Synthesis of ICG-Sar $_{5}$-(D-Leu-Aib) ${ }_{6}$-OMe (ICG-LP). ICG-LP (Fig. 5a) was synthesized according to Scheme S3 in the ESI. $\dagger$ A solution of the desalted compound of $\mathrm{H}$-(D-Leu-Aib) ${ }_{6}-\mathrm{OMe}$ $(6.57 \mathrm{mg}, 5.38 \mu \mathrm{mol})$ in distilled DMF $(50 \mu \mathrm{L})$ was added to a solution of Sar-NCA $(6.19 \mathrm{mg}, 53.8 \mu \mathrm{mol})$ in distilled DMF (150 $\mu \mathrm{L})$ under an Ar atmosphere, and the mixed solution was stirred at room temperature for $15 \mathrm{~h}$. After the complete consumption of Sar-NCA, ICG-sulfo-OSu (1.00 mg, $1.08 \mu \mathrm{mol})$ and DCC (0.44 $\mathrm{mg}, 2.15 \mu \mathrm{mol})$ were added to the solution in this order and stirred at room temperature for $25 \mathrm{~h}$ under an Ar atmosphere. The solvent was evaporated, and the residue was dissolved in DMF and purified using a Sephadex LH20 column. The chain length of the poly(sarcosine) was determined by the integration intensity ratio between $\mathrm{Sar} \mathrm{NCH}_{2}$ and Leu $\mathrm{CH}_{2}\left(\mathrm{CH}_{3}\right)_{2}$ in the ${ }^{1} \mathrm{H}$ NMR spectrum. The yield was determined by the absorbed light intensity of the ICG moiety in DMSO (absorption wavelength: $794 \mathrm{~nm}$ ). Yield: $2.33 \mathrm{mg}, 1.02 \mathrm{nmol}$ (95\%) (2 steps).

Preparation of molecular assemblies. The molecular assemblies were prepared by the injection method. ${ }^{10}$ 16RD (10 $\mathrm{mg}$ ) and the amphiphilic block polypeptides $(5 \mathrm{mg})$ were dissolved in ethanol $(100 \mu \mathrm{L})$, to prepare their stock solutions. Each mixed solution of the amphiphilic block-polypeptide solution $(2.5 \mu \mathrm{L})$ and the $16 \mathrm{RD}$ solution with the molar ratio of $0: 1,1: 1$, $2: 1,4: 1,8: 1,16: 1,24: 1$, and $32: 1$ was injected into a buffer $(0.5 \mathrm{~mL}, 10 \mathrm{mM}$ Tris-HCl, $\mathrm{pH} 7.4)$ with stirring at $4{ }^{\circ} \mathrm{C}$.

Preparation of ICG-labeled molecular assemblies. An ethanol solution $(10 \mu \mathrm{L})$ of $16 \mathrm{RD}(6.1 \mathrm{nmol}), \mathrm{A}_{3} \mathrm{~B}-\mathrm{LP}$ or $\mathrm{A}_{3} \mathrm{~B}$-apLP $(85.4 \mathrm{nmol})$, and ICG-LP $(1 \mathrm{nmol})$ in a molar ratio of $1: 14: 0.16$ was injected into saline $(0.5 \mathrm{~mL})$ kept in a sample vial at $4{ }^{\circ} \mathrm{C}$. This dispersion was kept stirring at $4{ }^{\circ} \mathrm{C}$ for $30 \mathrm{~min}$, allowed to reach room temperature, filtered through a membrane filter (polyethersulfone, $100 \mathrm{~nm}$ ), and then used for the fluorescent analysis and in vivo imaging experiment.

Transmission electron microscopy (TEM). TEM images were taken using a JEOL JEM-2000EXII at an accelerating voltage of $100 \mathrm{kV}$. A drop $(2 \mu \mathrm{L})$ of dispersion was mounted on a carboncoated $\mathrm{Cu}$ grid and stained negatively with $2 \%$ uranyl acetate, followed by the suction of the excess fluid with a filter paper.

Circular dichroism (CD). CD measurements were carried out using a JASCO J600 spectropolarimeter with an optical cell of 0.1 $\mathrm{cm}$ optical path length at room temperature.

Dynamic light scattering (DLS). The hydrodynamic diameter of the assemblies was measured by a DLS-8000KS (Photal Otsuka Electronics) using a He-Ne laser. Before DLS measurement, each 
prepared sample was filtered by a $0.20 \mu \mathrm{m}$ PVDF (polyvinylidene fluoride) syringe filter (GE Healthcare UK limited).

Cell culture. The pancreatic carcinoma (SUIT-2/pEF/LUC) cell line was maintained at $37{ }^{\circ} \mathrm{C}$ with $5 \%$ FBS (Nacalai Tesque, Inc. Kyoto, Japan) in Dulbecco's modified Eagle's medium (DMEM, Gibco, Invitrogen Corp. USA) supplemented with GlutaMAX ${ }^{\mathrm{TM}}-\mathrm{I}$ supplement $\left(2 \mathrm{mmol} \mathrm{L}^{-1}\right.$, Gibco, Invitrogen

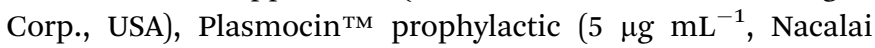
Tesque, Inc., Kyoto, Japan), penicillin (100 $\mathrm{U} \mathrm{mL}^{-1}$ ), and streptomycin (100 $\left.\mathrm{mg} \mathrm{mL}^{-1}\right)$.

In vivo near-infrared fluorescence (NIRF)-imaging with the ICG-labelled assemblies. SUIT-2/pEF/LUC cells $\left(5 \times 10^{5}\right.$ cells $)$ were dissolved in phosphate-buffered saline (PBS, $20 \mu \mathrm{L}$ ) and subcutaneously inoculated into the right femoral region of 7 week-old BALB/c nu/nu mice ( $n=4$ per group). The molecular assembly composed of $16 \mathrm{RD}$ and $\mathrm{A}_{3} \mathrm{~B}-\mathrm{LP}$ or $\mathrm{A}_{3} \mathrm{~B}$-apLP $(5 \mathrm{mg}$ $\mathrm{kg}^{-1}, 100 \mu \mathrm{L}$ ) was injected via the tail vein to the mice at 1 week after the tumor transplantation. The second dose of the ICGlabelled molecular assembly $\left(5 \mathrm{mg} \mathrm{kg}^{-1}, 100 \mu \mathrm{L}\right)$ was injected to the mice at 1 week after the first dose. The injected ICG amount was set to be $5 \mathrm{nmol} \mathrm{kg}{ }^{-1}$. NIRF images were taken at 15 min, $1 \mathrm{~h}, 3 \mathrm{~h}, 6 \mathrm{~h}, 9 \mathrm{~h}$, and $24 \mathrm{~h}$ after the second dose. During the imaging process, the mice were held on the imaging stage under anesthetized condition with $2.5 \%$ of isoflurane gas in the air flow (1.5 $\left.\mathrm{L} \mathrm{min}^{-1}\right)$. The pseudo images were constructed from the photon counts.

Ethics. All of our in vivo animal experiments were approved by the Animal Research Committee of Kyoto University. Animals were treated humanely.

\section{Acknowledgements}

This study is a part of translational research network program by MEXT, a part of S-innovation by JST, and a part of the innovative techno-hub for integrated medical bio-imaging of the project for developing innovation systems by MEXT.

\section{Notes and references}

1 J. Xie, S. Lee and X. Y. Chen, Adv. Drug Delivery Rev., 2010, 62, 1064.
2 Z. Wang, G. Niu and X. Y. Chen, Pharm. Res., 2014, 31, 1358.

3 T. Moore, H. Y. Chen, R. Morrison, F. L. Wang, J. N. Anker and F. Alexis, Mol. Pharmaceutics, 2014, 11, 24.

4 K. Y. Choi, G. Liu, S. Lee and X. Y. Chen, Nanoscale, 2012, 4, 330.

5 N. Ahmed, H. Fessi and A. Elaissari, Drug Discovery Today, 2012, 17, 928.

6 M. Yokoyama, J. Drug Targeting, 2014, 22, 576.

7 E. Hara, M. Ueda, C. J. Kim, A. Makino, I. Hara, E. Ozeki and S. Kimura, J. Pept. Sci., 2014, 20, 570.

8 H. Koide, T. Asai, K. Hatanaka, T. Urakami, T. Ishii, E. Kenjo, M. Nishihara, M. Yokoyama, T. Ishida, H. Kiwada and N. Oku, Int. J. Pharm., 2008, 362, 197.

9 T. Ishida, M. Harada, X. Y. Wang, M. Ichihara, K. Irimura and H. Kiwada, J. Controlled Release, 2005, 105, 305.

10 B. F. Lin, R. S. Marullo, J. F. Robb, D. V. Krogstad, P. Antoni, D. J. Hawker, L. M. Campos and M. V. Tirrell, Nano Lett., 2011, 11, 3946.

11 A. Uesaka, I. Hara, T. Imai, J. Sugiyama and S. Kimura, RSC Adv., 2015, 5, 14697.

12 H. Matsui, M. Ueda, A. Makino and S. Kimura, Chem. Commun., 2012, 48, 6181.

13 M. Ueda, A. Makino, T. Imai, J. Sugiyama and S. Kimura, Soft Matter, 2011, 7, 4143.

14 M. Ueda, A. Makino, T. Imai, J. Sugiyama and S. Kimura, Langmuir, 2011, 27, 4300.

15 M. Ueda, A. Makino, T. Imai, J. Sugiyama and S. Kimura, Polym. J., 2013, 45, 509.

16 A. Uesaka, M. Ueda, A. Makino, T. Imai, J. Sugiyama and S. Kimura, Langmuir, 2014, 30, 1022.

17 T. Kanzaki, Y. Horikawa, A. Makino, J. Sugiyama and S. Kimura, Macromol. Biosci., 2008, 8, 1026.

18 M. Ueda, A. Makino, T. Imai, J. Sugiyama and S. Kimura, J. Pept. Sci., 2011, 17, 94.

19 M. Ueda, A. Makino, T. Imai, J. Sugiyama and S. Kimura, Chem. Commun., 2011, 47, 3204.

20 P. K. Maiti, T. Cagin, G. F. Wang and W. A. Goddard, Macromolecules, 2004, 37, 6236.

21 S. Kimura, Org. Biomol. Chem., 2008, 6, 1143. 